\title{
PERFIL DOS ÓBITOS POR TUBERCULOSE PULMONAR EM UM MUNICÍPIO DO NORDESTE BRASILEIRO DURANTE O PERÍODO DE 2005-2014
}

\author{
Francisca Bárbara Gomes da Silva ${ }^{1}$ \\ Mariana Borges Sodré ${ }^{2}$ \\ Floriacy Stabnow Santos ${ }^{3}$ \\ Ana Cristina Pereira de Jesus Costa ${ }^{4}$ \\ Jaisane Santos Melo Lobato ${ }^{5}$ \\ Francisca Jacinta Feitoza de Oliveira ${ }^{6}$ \\ Marcelino Santos Neto ${ }^{7}$
}

SILVA, F. B. G. da; SODRÉ, M. B.; SANTOS, F. S.; COSTA, A. C. P. de J.; LOBATO, J. S. M.; OLIVEIRA, F. J. F. de; SANTOS NETO, M. Perfil dos óbitos por tuberculose pulmonar em um município do nordeste brasileiro durante o período de 2005-2014. Arq. Cienc. Saúde UNIPAR, Umuarama, v. 21, n. 3, p, 147-153, set./dez. 2017.

\begin{abstract}
RESUMO: Objetivou-se identificar o perfil dos óbitos por tuberculose pulmonar em um município do nordeste brasileiro durante o período de 2005-2014. Trata-se de um estudo descritivo, exploratório, documental, com abordagem quantitativa, realizado por meio do Sistema de Informação sobre Mortalidade na qual a amostra foi composta por 37 óbitos cuja causa básica foi por tuberculose pulmonar. O tratamento dos dados se deu por meio de estatística descritiva simples. Houve prevalência de óbitos com idade média de 60 anos, predomínio da forma pulmonar, sem menção de confirmação bacteriológica ou histológica $(81,0 \%)$, sexo masculino $(65,0 \%)$, raça/cor parda $(67,0 \%)$, estado civil solteiro (51,0\%), parcela predominante apresentou ensino médio completo $(32,0 \%)$ e aposentados $(32,0 \%)$. Observou-se ainda que a maioria dos óbitos ocorreu no hospital $(73,0 \%)$, com assistência médica antes do óbito $(76,0 \%)$, sendo a maior parcela atestada por médico assistente $(38,0 \%)$ e a maioria absoluta dos óbitos $(94,0 \%)$ não foi submetida à necropsia. A presente investigação revelou o perfil de uma população fatalmente atingida pela tuberculose pulmonar, evidenciando aspectos epidemiológicos importantes a serem considerados em termos de gestão e organização dos serviços de saúde para a equidade no acesso, desenvolvimento social e intensificação das ações de controle da doença.
\end{abstract}

PALAVRAS-CHAVE: Causa Básica de Morte. Registros de Mortalidade. Sistemas de Informação em Saúde. Tuberculose Pulmonar.

\section{PULMONARY TUBERCULOSIS DEATHS IN A CITY IN NORTHEAST BRAZIL FROM 2005 TO 2014}

\begin{abstract}
The purpose of this study was to identify the profile of pulmonary tuberculosis deaths in a city in the Brazilian Northeast during the period from 2005 to 2014. This is a descriptive, exploratory, documental study with a quantitative approach carried out through the Mortality Information System considering a sample of 37 deaths caused by pulmonary tuberculosis. Data were processed using simple descriptive statistics. Z prevalence of deaths with a mean age of 60 years old was observed, with predominance of the pulmonary form, with no mention of bacteriological or histological confirmation (81.0\%), males (65.0\%), mixed race/color $(67.0 \%)$, single (51.0\%), and a predominant part had a high school education (32.0\%) and were retired (32.0\%). It was also observed that most deaths occurred in hospital $(73.0 \%)$, with medical assistance before death $(76.0 \%)$, and the largest portion was assisted by an attending physician $(38.0 \%)$ and the absolute majority of deaths $(94.0 \%)$ were not submitted to necropsy. This investigation revealed the profile of a population fatally affected by pulmonary tuberculosis, evidencing important epidemiological aspects to be considered in terms of health service management and organization in order to provide equity in access, social development and intensification of disease control actions.
\end{abstract}

KEYWORDS: Health information systems. Mortality records. Mortality. Pulmonary tuberculosis. Underlying Cause of Death.

DOI: 10.25110 arqsaude.v21i3.2017.5847

'Acadêmica de Enfermagem do Centro de Ciências Sociais, Saúde e Tecnologia (CCSST) da Universidade Federal do Maranhão (UFMA). Avenida da Universidade S/N, Bom Jesus, Imperatriz-Ma, 65.900-000. (99) 3529-6062. Email: barbara.ufma@hotmail.com

${ }^{2}$ Acadêmica de Enfermagem do Centro de Ciências Sociais, Saúde e Tecnologia (CCSST) da Universidade Federal do Maranhão (UFMA). Avenida da Universidade S/N, Bom Jesus, Imperatriz-Ma, 65.900-000. (99) 3529-6062. Email: marianaborgessodre@hotmail.com

${ }^{3}$ Enfermeira. Doutora em Ciências - Saúde Pública - EERP/USP. Profa Adjunta II do Curso de Enfermagem do Centro de Ciências Sociais, Saúde e Tecnologia (CCSST) da Universidade Federal do Maranhão (UFMA). Avenida da Universidade S/N, Bom Jesus, Imperatriz-Ma, 65.900-000. (99) 35296062. Email: floriacys@gmail.com

${ }^{4}$ Enfermeira. Doutora em Enfermagem - UFC . Prof ${ }^{a}$ Adjunta I do Curso de Enfermagem do Centro de Ciências Sociais, Saúde e Tecnologia (CCSST) da Universidade Federal do Maranhão (UFMA). Avenida da Universidade S/N, Bom Jesus, Imperatriz-Ma, 65.900-000. (99) 3529-6062. Email: anacristina itz@hotmail.com

${ }^{5}$ Nutricionista. Mestre em Doenças Tropicais - UFPA. Prof ${ }^{a}$ Assistente I dos Cursos de Medicina e Enfermagem do Centro de Ciências Sociais, Saúde e Tecnologia (CCSST) da Universidade Federal do Maranhão (UFMA). Avenida da Universidade S/N, Bom Jesus, Imperatriz-Ma, 65.900-000. (99) 35296062. Email: jaisanelobato@hotmail.com

${ }^{6}$ Enfermeira. Mestre em Saúde Baseada em Evidências - UNIFESP . Prof ${ }^{a}$ Adjunta I do Curso de Enfermagem do Centro de Ciências Sociais, Saúde e Tecnologia (CCSST) da Universidade Federal do Maranhão (UFMA). Avenida da Universidade S/N, Bom Jesus, Imperatriz-Ma, 65.900-000. (99) 35296062. Email: jacinta_feitoza@hotmail.com

${ }^{7}$ Farmacêutico-bioquímico. Doutor em Ciências - Saúde Pública - EERP/USP. Prof ${ }^{\circ}$ Adjunto II do Curso de Enfermagem do Centro de Ciências Sociais, Saúde e Tecnologia (CCSST) da Universidade Federal do Maranhão (UFMA). Avenida da Universidade S/N, Bom Jesus, Imperatriz-Ma, 65.900-000. (99) 3529-6062. Email: marcelinosn@gmail.com 


\section{Introdução}

A tuberculose (TB), desde 1993, é considerada pela Organização Mundial de Saúde (OMS) como uma urgência mundial, e foi destacada como doença prioritária em vários países. No Brasil, um dos 22 países que concentram mais de $80 \%$ da carga bacilífera da doença, os indicadores epidemiológicos de incidência, prevalência e mortalidade ainda encontram-se elevados, especialmente em determinadas localidades consideradas prioritárias para o controle da doença, especialmente Índia, China e África do Sul são os países com maior carga da doença (World Health Organization [WHO], 2015).

Segundo Brasil (2016), entre os anos 2005 e 2014 foram registrados 26.297 indivíduos que tiveram o diagnóstico da doença confirmado. Destes, 18.861 indivíduos conseguiram a cura e 2.633 abandonaram o tratamento. Neste mesmo período, ocorreram 1.742 óbitos por TB, sendo que 1.415 foram confirmados como causa básica, conforme a Classificação Internacional de Doenças $n^{\circ} 10$ (CID-10): TB por via respiratória sem confirmação bacteriológica ou histológica (CID-10: A16) (WHO, 2015; BRASIL, 2016). Se os percentuais de cura para TB ainda estão baixos, muito provavelmente os pacientes por TB diagnosticados não estão concluindo o tratamento, o que favorece o desenvolvimento da TB, internações e casos de óbitos pela doença (WHO, 2015).

Dentre as formas clínicas da doença, a tuberculose pulmonar (TBP), além de ser mais frequente, é também a mais relevante para a saúde pública mundial, visto que se constitui como propagadora do bacilo entre as populações (ALAVI-NAINI et al., 2013). Diagnosticar e tratar, correta e prontamente, os casos de TBP são as principais medidas para o controle da doença. Nesse sentido, esforços devem ser realizados no sentido de encontrar precocemente o paciente e oferecer o tratamento adequado, interrompendo a cadeia de transmissão da doença (BRASIL, 2014).

A TBP demonstra relação direta com a pobreza e está associada à exclusão social e à marginalização de parte da população submetida às más condições de vida, como moradia precária, desnutrição, dificuldade de acesso aos serviços e bens públicos (SOUSA; PINHEIRO, 2011) e ainda faz parte da lista de causas de mortes evitáveis, haja vista que, se instituídas ações adequadas de promoção, proteção e recuperação da saúde dos indivíduos e famílias pelos sistemas locais de saúde, estes eventos não ocorreriam (MALTA et al., 2011).

Conhecer e entender os aspectos sociais, biológicos e operacionais da TBP sempre foi a principal meta dos estudiosos da doença. Hoje, mesmo com todo o avanço tecnológico no conhecimento científico, não é o suficiente para frear a disseminação do Mycobacterium tuberculosis, possibilitando cada vez mais novos casos da doença, internações evitáveis e óbitos inaceitáveis.

Pesquisas relacionadas à mortalidade por TBP vêm sendo estimuladas por serem consideradas como um importante instrumento para detecção de falhas dos sistemas de saúde (SELIG et al., 2004), uma vez que a investigação desse objeto permite traçar o perfil da TBP acompanhando o indivíduo em diferentes situações da doença, além de possibilitar análises adicionais sobre a vigilância dos casos e atendimentos dos pacientes (SOUSA; PINHEIRO, 2011).
Por meio de uma revisão da literatura realizada em bases indexadas, como Medical Literature Analysis and Retrival Sistem on-line (MEDLINE), Literatura Latino-Americana em Ciências da Saúde (LILACS) e Scientific Eletronic Library Online (SCIELO), pode-se verificar poucos estudos publicados no país, especificamente, sobre a mortalidade por TB (SANTOS NETO et al., 2014; MOTA et al., 2003; VICENTIN; SANTO; CARVALHO, 2002; YAMAMURA et al., 2015; LINDOSO et al., 2008; SELIG et al., 2004). Desses estudos, nenhum deles abordou o cenário de Imperatriz/MA, um dos 177 municípios prioritários para o controle da doença em nível nacional por apresentar critérios de priorização, tais como coeficiente de incidência superior a 47/100.000 habitantes e coeficiente de mortalidade superior a 3/100.000 habitantes (BRASIL, 2014).

Com relação aos indicadores sociais e econômicos, o município possui expectativa de vida ao nascer de 73,2 anos; índice de desenvolvimento humano (IDH) de 0,73 e índice de exclusão social (IES) em torno de 0,6; incidência de pobreza de 55,28\%; e Índice de Gini de 0,46 (IBGE, 2016).

Considerando a magnitude e a complexidade que envolve a ocorrência de óbitos por TBP, circunscritos à necessidade de compreender sua dinâmica no cenário em questão para intervenções em saúde mais efetivas e direcionadas às populações mais vulneráveis, é que se propôs este estudo que tem como objetivo identificar o perfil dos óbitos por tuberculose pulmonar em Impertatriz-MA durante o período de 2005-2014.

\section{Material e Método}

Trata-se de um estudo descritivo, exploratório, documental, com abordagem quantitativa, na qual utilizou os registros de óbitos por TBP do município de Imperatriz/MA (Figura 1), localizado no sudoeste do Estado do Maranhão, região Nordeste do Brasil, que possui uma área territorial de $1.368,987 \mathrm{~km}^{2}$ e uma população estimada de 252.320 habitantes (IBGE, 2016).

O município está localizado a $626 \mathrm{~km}$ da capital São Luís, e é considerado o segundo maior centro populacional, econômico, político e cultural do Maranhão. A cidade conta com apenas $23 \%$ de rede de esgoto,tem $86 \%$ de abastecimento de água potável possui taxa de analfabetismo de $9,7 \%$.

Figura 1: Mapa do Brasil, com destaque para o estado do Maranhão e para a cidade de Imperatriz.

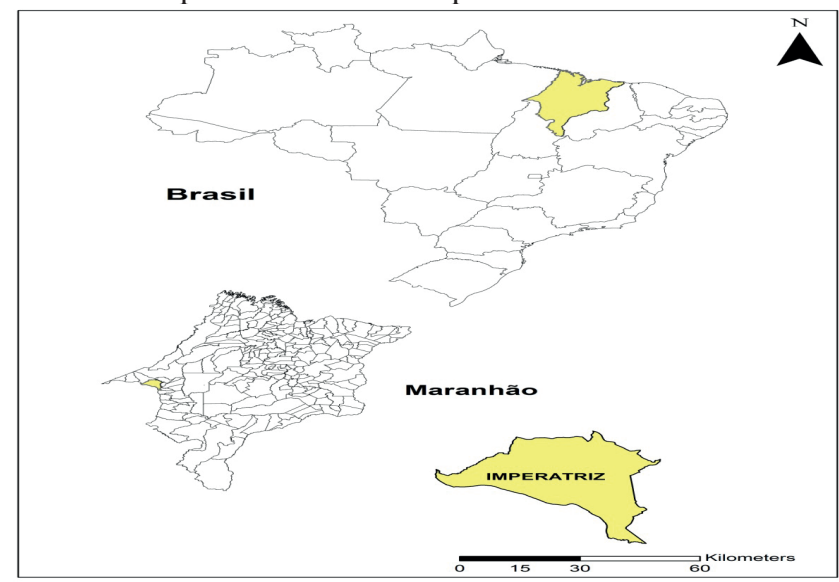

Fonte: Adaptado de bases geográficas (IBGE, 2016). 
A população do estudo foi composta por todos os casos de óbitos que tiveram como causa básica a TBP de acordo com CID-10 de A15.0 a A15.3 e de A16.0 a A16.2, registrados no Sistema de Informação sobre Mortalidade (SIM) da Vigilância em Saúde da Secretaria Municipal de Saúde (SEMUS) de Imperatriz/MA no período de 2005 a 2014. É válido ainda mencionar que foram considerados para a pesquisa os casos de óbitos de residentes tanto da zona urbana quanto rural do município.

Foram excluídos os óbitos de indivíduos residentes em outras localidades e aqueles que apresentassem outra forma clínica que não a pulmonar. Ressalta-se que óbitos de coinfecção TB/HIV (CID B20.0) não foram considerados neste estudo devido a complexidade patológica dos casos e por não serem considerados como óbito por TB no encerramento das notificações.

Os dados foram coletados em agosto de 2016 e as variáveis de interesse do presente estudo foram obtidas via declaração de óbito (DO) utilizado no Brasil e compreenderam características socioeconômicas e demográficas, como idade, sexo, raça/cor, Estado civil, escolaridade e ocupação, além de variáveis operacionais, tais como local de ocorrência do óbito, assistência médica, necropsia, causa básica e médico responsável pelo atestado de óbito. Ressalta-se que tais variáveis foram coletadas diretamente do SIM, por meio do qual foi gerado um arquivo em planilha Excell para realização das análises propostas.

No período compreendido entre 2005 e 2014, foram identificados 37 óbitos por TBP, sendo $30(81,0 \%)$ referentes à "tuberculose pulmonar, sem menção de confirmação bacteriológica ou histológica" (CID-10 A16.2); três (8,0\%) à "tuberculose pulmonar, com confirmação por exame microscópico da expectoração com ou sem cultura" (CID-10 A15.0); um $(3,0 \%)$ à "tuberculose pulmonar, com confirmação por meio não especificado" (CID-10 A15.3); e um (8,0\%) à "tuberculose pulmonar, sem realização de exame bacteriológico ou histológico" (CID-10 A16.1) junto ao SIM da SEMUS de Imperatriz/MA.

A análise desses dados foi realizada por meio da estatística descritiva dos parâmetros quantitativos utilizando o programa Statistical Package for the Social Sciences (SPSS), versão 17.0, sendo expressas frequências absolutas e percentuais para as variáveis categóricas. Em relação à variável idade, os indivíduos que evoluíram para óbito foram categorizados a partir da mediana da idade, sendo classificados, portanto, por idade acima ou abaixo do valor obtido. Utilizou-se a mediana da idade dos indivíduos que faleceram por TBP por ser a medida de tendência central que fornece uma ideia melhor de um valor típico porque não é tão distorcida por valores extremamente altos ou baixos (CALLEGARI-JACQUES, 2003).

A média de idade dos indivíduos que evoluíram para óbito por TBP no período analisado foi de 60 anos, desvio padrão (DP) 16,0 e a mediana foi de 60 anos. O indivíduo mais novo possuía 22 e o mais velho 93 anos.

O estudo obedeceu aos preceitos éticos estabelecidos pela Resolução n 466/2012, do Conselho Nacional de Saúde (CNS) na qual aprova a realização de pesquisa envolvendo seres humanos. O projeto de pesquisa foi apreciado e aprovado pelo Comitê de Ética em Pesquisa da Universidade Federal do Maranhão (CEP UFMA), sob parecer $n^{\circ} 1.140 .668$, de 29 de junho de 2015, Certificado de Apresentação para Apreciação Ética (CAAE) no 44678415.9.0000.5087.

\section{Resultados}

A tabela 1 apresenta as características socioeconômicas e demográficas dos indivíduos. Houve prevalência de óbitos na qual ocorreram em indivíduos com idade menor ou igual a 60 anos (54\%); sexo masculino $(65,0 \%)$; raça/cor parda $(67,0 \%)$; e solteiros $(51,0 \%)$. Em relação à escolaridade, predominou o ensino médio completo $(32,0 \%)$ e ocupação de aposentado ou pensionista $(32,0 \%)$.

Tabela 1: Características socioeconômicas e demográficas dos casos de óbitos por TBC no período de 2005-2014. Imperatriz (MA), 2015.

\begin{tabular}{|c|c|c|}
\hline Variáveis & $\mathbf{n}$ & $\%$ \\
\hline \multicolumn{3}{|l|}{ Idade } \\
\hline$\leq 60$ anos & 20 & 54,0 \\
\hline$>60$ anos & 17 & 46,0 \\
\hline \multicolumn{3}{|l|}{ Sexo } \\
\hline Masculino & 24 & 65,0 \\
\hline Feminino & 13 & 35,0 \\
\hline \multicolumn{3}{|l|}{ Raça/cor } \\
\hline Branca & 07 & 19,0 \\
\hline Preta & 04 & 11,0 \\
\hline Amarela & 01 & 3,0 \\
\hline Parda & 25 & 67,0 \\
\hline \multicolumn{3}{|l|}{ Estado Civil } \\
\hline Solteiro & 19 & 51,0 \\
\hline Casado & 11 & 30,0 \\
\hline Viúvo & 06 & 16,0 \\
\hline Divorciado & 01 & 3,0 \\
\hline \multicolumn{3}{|l|}{ Escolaridade } \\
\hline Sem escolaridade & 02 & 6,0 \\
\hline $1^{\mathrm{a}} \mathrm{a} 4^{\mathrm{a}}$ série & 09 & 24,0 \\
\hline $5^{\mathrm{a}}$ a $8^{\mathrm{a}}$ série & 09 & 24,0 \\
\hline Ensino médio & 12 & 32,0 \\
\hline Superior incompleto & 05 & 14,0 \\
\hline \multicolumn{3}{|l|}{ Ocupação } \\
\hline Serviços gerais & 05 & 13,0 \\
\hline Garimpeiro & 01 & 3,0 \\
\hline Dona de casa & 04 & 10,0 \\
\hline Aposentado/Pensionista & 12 & 32,0 \\
\hline Motorista & 01 & 3,0 \\
\hline Trabalhador agropecuário & 08 & 22,0 \\
\hline Artesã & 01 & 3,0 \\
\hline Pescador artesanal & 01 & 3,0 \\
\hline Comerciante & 03 & 8,0 \\
\hline Ignorado & 01 & 3,0 \\
\hline Total & 37 & 100,0 \\
\hline
\end{tabular}


Com relação aos indicadores operacionais apresentados na tabela 2, observou-se que $94,0 \%$ dos casos de óbitos não foram submetidos à necropsia e, ainda, que 73,0\% destes ocorreram em hospital e 76,0\% dos pacientes receberam assistência médica antes do óbito. Quanto ao responsável pelos registros, predominou o médico assistente $(38,0 \%)$.

Tabela 2: Características operacionais dos casos de óbitos por TBC durante o período de 2005-2014. Imperatriz (MA), 2015.

\begin{tabular}{lcc}
\hline Variáveis & n & \% \\
\hline Local do Óbito & 27 & 73,0 \\
Hospital & 10 & 27,0 \\
Domicílio & & \\
Assistência Médica & 28 & 76,0 \\
Sim & 08 & 21,0 \\
Não & 01 & 3,0 \\
Ignorado & & \\
Necropsia & 35 & 94,0 \\
Não & 01 & 3,0 \\
Sim & 01 & 3,0 \\
Ignorado & & \\
Óbito atestado por médico & 14 & 38,0 \\
Assistente & 10 & 27,0 \\
Substituto & 11 & 30,0 \\
SVO* & 02 & 5,0 \\
IML** & $\mathbf{3 7}$ & $\mathbf{1 0 0 , 0}$ \\
\hline Total & & \\
\hline *SVO Serviço Vigiância & & \\
\hline
\end{tabular}

*SVO: Serviço de Vigilância do Óbito

**IML: Instituto Médico Legal

\section{Discussão}

Cabe inicialmente ressaltar que apesar da forma clínica pulmonar da TB ser facilmente diagnosticada, tratável e curável há mais de 50 anos gratuitamente via Sistema Único de Saúde (SUS), verifica-se ainda em cenário nacional o maior número de casos de TBP (BRASIL, 2016) e que óbitos pela doença estão entre as causas de morte dita como evitáveis e inaceitáveis do ponto de vista da justiça social (MALTA et al, 2011). Ademais, a TBP continua sendo a doença infecciosa que mais mata, mesmo que os fundamentos científicos para o diagnóstico e controle na comunidade sejam conhecidos há muito tempo.

As fontes de infecção encontram-se, principalmente, entre os doentes bacilíferos, responsáveis pela cadeia epidemiológica de transmissão, que ocorre, na grande maioria das vezes, por via aerógena, em ambientes fechados por meio de contatos íntimos e prolongados (LÖNNROTH, et al., 2010). Esses pacientes apresentam habitualmente tosse e expectoração por mais de três semanas e são chamados de sintomáticos respiratórios que, em conjunto com pacientes considerados suspeitos à radiografia de tórax e com os indivíduos contatos de pacientes bacilíferos, formam a base para a descoberta de casos de TB por meio da demanda espontânea aos serviços de saúde (BRASIL, 2014).
Evidenciou-se neste estudo um número expressivo de óbitos que foram registrados com CID A16.2 como causa básica, tal como em estudos realizados em diferentes cenários como Campo Grande/MS (ESPINDOLA,2010), no Estado do Rio de Janeiro (SELIG et al., 2004), no município de São Luís/MA (SANTOS NETO et al., 2014) e no município de Ribeirão Preto/SP (YAMAMURA et al., 2015) que apresentaram percentuais acima de $50 \%$ de óbitos pelo referido CID A16.2, sugerindo, assim, uma questão a ser resolvida pelos serviços e sistemas de saúde dos cenários citados e um desafio a ser superado sobretudo pela possibilidade de existência de falsos positivos entre os óbitos que tiveram como causa básica a TBP, uma vez que a maioria estava sem menção de confirmação bacteriologia ou histológica.

Tais resultados que destacam registros referentes à forma clínica pulmonar da TB remetem a uma questão problemática para os sistemas de saúde, pois essa forma clínica apresenta alto índice de letalidade e está entre a principal forma de transmissão da doença, o que suscita reflexões quanto às políticas públicas no que se refere à acessibilidade dos pacientes aos serviços públicos de saúde para diagnóstico e tratamento em tempo oportuno, bem como repercurte na necessidade de realizar busca ativa de pacientes sintomáticos respiratórios (SELIG et al., 2010). Alguém que recebe um diagnóstico tardio tem grande risco de morrer por TB, como evidencia a literatura, haja vista que a doença pode se espalhar por todo o pulmão e ainda acometer outras partes do organismo (RODRIGUEZ, 2009).

Vale destacar que a TBP continua sendo mundialmente um importante problema de saúde (WHO, 2015), exigindo o desenvolvimento de estratégias para o seu controle, considerando aspectos humanitários, econômicos e de saúde pública e tem levado mais indivíduos ao óbito que qualquer outra doença infecciosa e entre os doentes infectados pelo vírus da Acquired Immuno Deficiency Syndrome (AIDS) é a maior causa de morte, mesmo quando utilizado o tratamento com antirretroviral (BRASIL, 2014).

Analisando-se as características socioeconômicas e demográficas, o estudo apontou que $65 \%$ dos casos de óbitos por TBP no cenário em questão ocorreu em pacientes do sexo masculino, ocorrência justificável pelo fato de que homens em idade ativa são mais acometidos pela doença do que mulheres, conforme diversos estudos em diferentes cenários (ALAVI-NAINI et al., 2013; ÁLVAREZ et al., 2011; AUGUSTO et al., 2013; CALIARI; FIGUEIREDO, 2012; CECILIO et al., 2013; ESPINDOLA, 2010; HINO et al., 2007; JUNG et al., 2010; KAYIGAMBA et al., 2013; LINDOSO et al., 2008; MOREIRA et al., 2008; OLIVEIRA et al., 2013; YAMAMURA et al., 2015; SANTOS NETO et al., 2014; BRASIL, 2016). Atribui-se, possivelmente, ao fato dos homens estarem mais presentes no mercado de trabalho, menos presentes nos serviços de saúde e apresentarem maior prevalência de infecção por HIV, de alcoolismo e de usos abusivo de drogas (FAÇANHA, 2006), embora essas variáveis não tenham sido exploradas na presente pesquisa.

Evidenciou-se que os óbitos por TBP ocorreram em igual proporção entre indivíduos com idade menor ou igual a 60 anos, reiterando, portanto, com resultados encontrados em estudos realizados no Brasil, em cidades dos Estados de São Paulo e Salvador (OLIVEIRA; MARÍN-LEÓN; CARDOSO, 2004; LINDOSO et al., 2008; MOTA et al., 2003). 
Observou-se, ainda, a ocorrência de casos de óbitos TBP em idosos, uma vez que a idade máxima dos óbitos foi acima de 90 anos, também apresenta suas particularidades e encontra nessa população uma marcante susceptibilidade que está relacionada, principalmente, à diminuição da imunidade celular, afetada pelo processo de envelhecimento imunológico, depressão das defesas orgânicas e alterações na função imune e pulmonar (OLIVEIRA et al., 2005; CAVALCANTI et al., 2006). Outros fatores que contribuem de forma decisiva para o desenvolvimento da TBP em idosos são as inúmeras condições imunossupressoras associadas, tais como diabetes, insuficiência hepática, insuficiência renal, desnutrição e terapia prolongada com corticosteróides (CANTALICE FILHO; SANT'ANNA; BÓIA, 2007; OLIVEIRA et al., 2005; DOOLEY; CHAISSON, 2009).

$\mathrm{Na}$ presente investigação, observou-se ainda que o estado civil que se destacou dentre os casos de óbitos foi o de solteiro, corroborando com estudos realizados no município São Luís, no estado de Minas Gerais e Rio de Janeiro (ESPINDOLA, 2010; SANTOS NETO et al., 2014; SELIG et al., 2004 e YAMAMURA et al., 2015).

Quanto a raça/cor, os resultados obtidos expressam equivalência ao estudo realizado em Campo Grande (ESPINDOLA, 2010), São Luís (SANTOS NETO, et al., 2014) e demais municípios prioritários do Mato Grosso do Sul (LARROQUE, 2011) para o controle da TB, denotando que a maior parte dos óbitos ocorreu em indivíduos raça/cor parda, em consonância com o problema de morbidade e mortalidade no país (BRASIL, 2016; OLIVEIRA et al., 2013) e divergindo dos estudos realizados no Estado de São Paulo (LINDOSO et al., 2008 e YAMAMURA et al., 2015) onde a raça/cor branca foi a prevalente.

Os dados de escolaridade e ocupação encontrados nesta pesquisa foram divergentes com alguns estudos (AUGUSTO et al., 2013; ESPINDOLA, 2010; LARROQUE, 2011; LINDOSO et al., 2008 e SANTOS NETO et al., 2014 e BRASIL, 2016) na qual os mesmos relatam que a inexistência ou baixa escolaridade são evidenciadas como fatores de risco para a TBP e podem ser consideradas reflexos de um conjunto de condições socioeconômicas precárias que aumentam a vulnerabilidade à TBP e são responsáveis pela maior incidência dessa enfermidade, além de contribuírem para a não adesão ao tratamento e para o aumento da taxa de abandono do tratamento, bem como para a ocorrência da hospitalização e óbito por TB (OLIVEIRA et al., 2012).

Especificamente quanto à variável "ocupação", vale ressaltar que indivíduos em situações menos privilegiadas do ponto de vista financeiro, apresentaram maiores chances de desenvolver a TBP (AUGUSTO et al., 2013; ESPINDOLA, 2010; LARROQUE, 2011; LINDOSO et al., 2008 e SANTOS NETO et al., 2014). No presente estudo encontrou-se que os óbitos prevaleceram em indivíduos com Ensino Médio completo com ocupação de destaque para aposentados ou pensionistas. Ademais, o número prevalente da ocupação de aposentados ou pensionistas nesta casuística evidencia a vulnerabilidade das pessoas idosas a essa tão problemática patologia (BRASIL, 2016).

No tocante as características operacionais, 73,0\% dos óbitos analisados ocorreram no hospital. Essa situação assemelha-se com estudos nacionais realizados nos Estados do Rio de Janeiro (SELIG et al., 2004), São Paulo/SP (LIN-
DOSO et al., 2008), Mato Grosso do Sul (LARROQUE, 2011), Ribeirão Preto/SP (YAMAMURA et al., 2015) e ainda no município de São Luís/MA (SANTOS NETO et al., 2014) com registros iguais ou superiores a $80,0 \%$. Estudo de coorte de base populacional em país e da América Latina, China e Índia (FERRI et al., 2012) também revelou que a maioria dos óbitos ocorreu no hospital. Perrechi e Ribeiro (2009) apontam que apesar do diagnóstico da TBP ser relativamente simples, verifica-se, ainda, que grande parte dos casos é diagnosticada em hospitais o que representa custos elevados para o sistema de saúde e sequelas aos doentes (LÖNNROTH, et al., 2010).

Nessas localidades, a evolução para óbitos de paciente hospitalizados por TBP sugere dificuldade da Atenção Primária à Saúde (APS) na gestão, na oferta de recursos diagnósticos ou no manejo dos casos e no sistema de referência aos outros serviços de saúde (SELIG et al., 2010). Há também outro fator que ajuda a explicar esse resultado, que se refere ao abandono do tratamento, sendo ele um preditor para o desenvolvimento de multidroga resistência (MDR) e assim para as hospitalizações (KAYIGAMBA et al., 2013).

Nesse sentido, torna-se relevante a reestruturação e fortalecimento das ações de controle da TBP, com priorização da baciloscopia de escarro e teste molecular rápido para o diagnóstico, controle e monitoramento da doença, bem como o aprimoramento dos registros e dos Sistemas de Informações em Saúde (SIS) (ARCÊNCIO; OLIVEIRA; VILLA, 2007; OLIVEIRA et al., 2013).

Quanto a variável operacional assistência médica, $76,0 \%$ dos óbitos receberam essa assistência, concordando com outros estudos (SANTOS NETO et al., 2014; YAMAMURA et al., 2015) que a maioria dos óbitos também obtiveram assistência médica antes do óbito. No que diz respeito à confirmação dos casos de óbitos por TB por meio da realização da necropsia, evidenciou-se que $94,0 \%$ não realizaram este exame, o que exprime exatamente a fragilidade e insuficiência dos sistemas e serviços de saúde na redução das iniquidades sociais em saúde, conforme evidenciado por Curtis (2009) ao enfatizar a dimensão da Geografia da TB "uso e acesso aos serviços de saúde". Nesse sentido, torna-se preponderante a necessidade de uma reestruturação, bem como fortalecimento das ações de controle da TB na APS para que a mesma seja capaz de resolver, pelo menos $85 \%$ dos problemas de saúde da comunidade (VIACAVA et al., 2012).

Em se tratando dos agravos de notificação compulsória, como a TBP, em destaque neste estudo, a utilização de dados disponíveis no sistema de informação em saúde permite o acompanhamento do problema, colaborando para identificação de aspectos relevantes e incentivando a busca de novas intervenções para o controle da doença (CALIARI; FIGUEIREDO, 2012).

Cabe mencionar que o SIM, como fonte de informações para o estudo dos óbitos de uma determinada região, tem suas fragilidades. Uma delas é a própria subnotificação no país (ESPINDOLA, 2010; OLIVEIRA et al., 2012) que é uma das consequências da iniquidade do acesso aos serviços de saúde. Destacam-se também, como fragilidade, as lacunas no preenchimento dos registros, que são informações relevantes à gestão e ao planejamento em saúde. Nesta perspectiva, melhorar a qualidade dos registros em relação ao preenchimento dos campos e à atualização dos dados pro- 
venientes dos SIS são primordiais para a confiabilidade da análise epidemiológica (SANTOS NETO et al., 2014).

\section{Conclusão}

A presente investigação revelou o perfil de uma população fatalmente atingida pela TBP, evidenciando aspectos epidemiológicos importantes a serem considerados em termos de gestão e organização dos serviços de saúde para a equidade no acesso, desenvolvimento social e intensificação das ações de controle da doença.

O estudo, sem dúvida, traz importantes contribuições para o avanço do conhecimento na temática ao evidenciar que os óbitos por TBP são eventos socialmente determinados, reforçando a importância da proteção social por sistemas universais de saúde com vistas à eliminação da TB e não mais mortes injustas ou evitáveis.

Cabe ainda mencionar que os desafios para controlar a TB no cenário sob investigação e no Brasil persistem, e que as perspectivas para o controle da apenas se concretizarão se mais esforços forem lançados pelas três esferas de gestão do SUS, assim como se houver o estabelecimento de parcerias fora desse setor. Desse modo, são necessárias melhorias da qualidade do serviço prestado aos pacientes com $\mathrm{TB}$, garantindo-lhes o direito à prevenção, ao diagnóstico correto e oportuno da doença e ao tratamento adequado e humanizado.

Por fim, dados inconsistentes relacionados às variáveis investigadas constituíram-se fatores limitantes deste estudo, visto que foram encontrados registros como "ignorados" ocasionando viés de informação.

\section{Agradecimentos}

À Fundação de Amparo à Pesquisa e ao Desenvolvimento Científico e Tecnológico do Maranhão (FAPEMA) - Processo Universal 0562/2015 e BIC - 03198/15 pelo apoio financeiro para realização da pesquisa e à Vigilância em Saúde da Secretaria Municipal de Saúde (SEMUS) de Imperatriz/MA pela concessão dos dados.

\section{Referências}

ALAVI-NAINI, R. et al. Factors associated with mortality in tuberculosis patients. Journal of Research in Medical Sciences. Isfahan, v. 18, n. 1, p. 52-55, 2013.

ÁLVAREZ, J. L. et al. Educational inequalities in tuberculosis mortality in six teen European populationns. International Journal of Tuberculosis and Lung Disease. Paris, v. 15, n. 11, p. 1461-1467, 2011

ARCÊNCIO, R. A.; OLIVEIRA, M. F.; VILLA, T. C. S. Internações por tuberculose pulmonar no Estado de São Paulo no ano de 2004. Ciência \& Saúde Coletiva. Rio de Janeiro, v. 12, n. 2, p. 409-417, 2007.

AUGUSTO, C. J. et al. Características da tuberculose no estado de Minas Gerais entre 2002 e 2009. Jornal Brasileiro de Pneumologia. Brasília, v. 39, n. 3, p. 357364, 2013.
BRASIL. Ministério da Saúde. Secretaria de Vigilância em Saúde. Departamento de Vigilância das Doenças Transmissíveis. Panorama da tuberculose no Brasil: indicadores epidemiológicos e operacionais. Brasília, 2014. $92 \mathrm{p}$.

BRASIL. Ministério da Saúde. Portal Saúde: indicadores epidemiológicos da tuberculose. 2016. Disponível em: $<$ http://portalms.saude.gov.br/saude-de-a-z/tuberculose $>$. Acesso em: 29 jun. 2016.

\section{CALLEGARI-JACQUES, S. M. Bioestatística. Princípios e aplicações. Porto Alegre, Artmed, 2003.}

CALIARI, J. S.; FIGUEIREDO, R. M. Tuberculosis: patient profile, service flowchart, and nurses opinions. Acta Paulista de Enfermagem, São Paulo, v. 25, n. 1, p. 43-47. 2012.

CANTALICE, F.; SANT’ANNA, C. C.; BÓIA, M. N. Aspectos clínicos da tuberculose pulmo nar em idosos atendidos em hospital universitário do Rio de Janeiro, RJ, Brasil. Jornal Brasileiro de Pneumologia, Brasília-DF, v. 33, n. 6, p. 699-706, 2007.

CAVALCANTI, Z. R. et al. Características da tuberculose em idosos no Recife (PE): contribuição para o programa de controle. Jornal Brasileiro de Pneumologia. Brasília-DF, v. 32, n. 6, p. 535-543, 2006.

CECILIO, H. P. M. et al. Perfil das internações e óbitos hospitalares por tuberculose. Acta Paulista de Enfermagem, São Paulo, v. 26, n. 3, p. 250-255, 2013.

CURTIS, S. Health and Inequality: Geographical Perspectives. Sage Publications, 2009. 329 p.

DOOLEY, K. E.; CHAISSON, R. E. Tuberculosis and diabetes mellitus: convergence of two epidemics. The Lancet Infectious Diseases, USA, v. 9, n. 12, p. 737-46, 2009.

ESPINDOLA, L. C. D. Estudo da mortalidade por tuberculose em Campo Grande - MS, 2001 a 2008. 2010. 44 f. Dissertação (Mestrado Profissional em Saúde Pública). Escola Nacional de Saúde Pública Sergio Arouca da Fiocruz, Rio de Janeiro, 2010.

FAÇANHA, M. C. Evolução da mortalidade por tuberculose em Fortaleza (CE), entre 1980 e 2001. Jornal Brasileiro de Pneumologia, Brasília, v. 32, n. 6, p. 553558, nov/dez. 2006

FERRI, C. P. et al. Socioeconomic Factors and All Cause and Cause-Specific Mortality amon g Older People in Latin America, India, and China: A Population-Based Cohort Study. PLOS Medicine, San Francisco, v. 9, n. 2, p. 114, fev. 2012.

HINO, P. et al. Contr ole da tuberculose na perspectiva da vigilância da saúde. Esc Anna Nery (impr.). 2007; 
$15(2): 417-421$.

IBGE. Instituto Brasileiro de Geografia e Estatística. Mapas: bases e referenciais. Rio de Janeiro: IBGE, 2016.Disponível em < http://mapas.ibge.gov.br/bases-ereferenciais/basescartograficas/cartas $>$. Acesso em: 23 jun. de 2016.

JUNG, R. S. et al. Trends in tuberculosis mortality in the United States, 1990-2006: a populati on-based case-control study. Public Health Reports, Washington, v. 125, n. 3, p.389-397, maio/jun. 2010.

KAYIGAMBA, F. R. et al. Adherence to Tuberculosis Treatment, Sputum Smear Conversion and Mortality: A Retrospective Cohort Study in 48 Rwandan Clinics. PLOS Medicine, San Francisco, v. 8, n. 9, set. 2013.

LARROQUE, M. M. Mortalidade por tuberculose em municípios prioritários do estado de Mato Grosso do Sul, 1999-2008. 2011. 86 f. Dissertação (Mestrado em Doenças Infecciosas e Parasitárias). Universidade Federal do Mato Grosso do Sul, Mato Grosso do Sul, 2011.

LINDOSO, A. A. B. P. et al. Perfil de pacientes que evoluem para óbito por tuberculose no município de São Paulo, 2008. Cadernos de Saúde Pública, Rio de Janeiro, v. 42 , n. 5 , p. $805-812$, out. 2008.

LÖNNROTH, K. et al. Tuberculosis control and elimination 2010-50: cure, care, and social development. The Lancet, Cidade do Cabo, v. 375, p. 1814-1829, 2010

MALTA, D. C. et al. Atualização da lista de causas de mortes evitáveis ( 5 a 74 anos de idade) por intervenções do Sistema Único de Saúde do Brasil. Epidemiologia e Serviços de Saúde. Brasília, v. 20, n. 3, p. 409-412, jul/ set.2011.

MOREIRA, C. M. M. et al. Mortalidade específica por tuberculose no estado do Espírito Sant o, no período de 1985 a 2004. Jornal Brasileiro de Pneumologia. Brasília, v. 34, n. 8, p. 601-606, ago. 2008.

MOTA, F. F. et al. Distribuição espacial da mortalidade por tuberculose em Salvador, Bahia, Brasil. Cadernos de Saúde Pública, Rio de Janeiro, v. 19, n. 4, p. 915-922, 2003.

OLIVEIRA, H. B.; MARÍN-LEÓN, L.; CARDOSO, J. C. Perfil de mortalidade de pacientes com tuberculose relacionada à comorbidade tuberculose-Aids. RevSaúde Pública. 2004; 38(4): 503-10.

OLIVEIRA, M. A. et al. Comparando estruturas sociais no Rio de Janeiro e em São Paulo. Dados. Rio de Janeiro. v. 51, n. 1, p. 215-238, 2005.

OLIVEIRA, G. P. et al. Uso do sistema de informação sobre mortalidade para identificar subnotificação de casos de tuberculose no Brasil. Revista Brasileira de
Epidemiologia, São Paulo, v. 15, n. 3, p. 468-477, 2012.

Tuberculosis in Brazil: last ten years analysis 2001-2010. Brazilian Society of Infectious Diseases, Salvador, v. 17 n. 2, p. 218-233, mar/abr. 2013.

PERRECHI, M. C. T.; RIBEIRO, S. A. Tratamento de tuberculose: integração entre assistênci a hospitalar e rede básica na cidade de São Paulo. Jornal Brasileiro de Pneumologia, Brasília - DF, v. 35, n. 11, p. 1100-1106, 2009.

RODRIGUEZ, D. Death From a Preventable Disease: Tuberculosis. Everyday Health, 2009. Disponível em: https://www.everydayhealth.com/tuberculosis/death-from-apreventable-disease.aspx.

SANTOS NETO, M. et al. Análise espacial dos óbitos por tuberculose pulmonar em São Luís (MA). J Bras Pneumol, 2014; 40: 543-551.

SELIG, L. et al. Óbitos atribuídos à tuberculose no Estado do Rio de Janeiro. Jornal Brasileiro de Pneumonia, Brasília, v. 30, n. 4, p. 417-424, jul./ago. 2004.

SELIG, L. et al. Proposta de vigilância de óbitos por tuberculose em sistemas de informação. Revista de Saúde Pública, São Paulo, v. 44, n. 6, p. 1072-1078, dez. 2010.

SOUSA, L. M. O.; PINHEIRO, R. S. Óbitos e internações por tuberculose não notificados no município do Rio de Janeiro. Revista de Saúde Pública, São Paulo, v. 45, n. 1, p. 31-39. Fev. 2011.

VIACAVA, F. et al. Avaliação de Desempenho de Sistemas de Saúde: um modelo de análise. Ciência \& Saúde Coletiva, Rio de Janeiro, v.17, n. 4, p. 921-934, 2012.

VICENTIN, G.; SANTO, A. H.; CARVALHO, M. S. Mortalidade por tuberculose e indicadores sociais no município do Rio de Janeiro. Ciência \& Saúde Coletiva. Rio de Janeiro, v. 7, n. 2, p. 253-263, 2002.

\section{WORLD HEALTH ORGANIZATION. Global} tuberculosis report 2015. Geneva: WHO 2015. Disponível em: <http://www.who.int/tb/publications/global_report/ en/>. Acesso em: 16 Julho de 2016.

YAMAMURA, M. et al. Características epidemiológicas dos casos de óbito por tuberculose e territórios vulneráveis. Rev. Latino-Am. Enfermagem. Ribeirão Preto, 23(5): 9108, set.-out. 2015. 